\title{
A STUDY OF CURVED SURFACES BY MEANS OF CERTAIN ASSOCIATED RULED SURFACES*
}

\author{
BY \\ P. O. BELL
}

Introduction. In this paper a point correspondence is introduced which is proving to be very helpful in the study of a general non-ruled analytic surface in ordinary space. If on a surface $S$ tangents to the curves of an asymptotic family are constructed at the points of two curves of $S$ which are not members of the family and which intersect in a point $y$ of $S$, two ruled surfaces are thereby formed which have at $y$ a common generator. The plane which is tangent to one of these ruled surfaces at a selected point of the common generator is tangent to the other at a distinct point whose location depends on the selection of the first point and on the choice of the two curves which determine the ruled surfaces. The use of this correspondence serves the following fourfold purpose: (1) to unify many of the apparently isolated topics which have been studied heretofore, (2) to interpret geometrically, by methods which are simpler than those formerly used, quantities which are intrinsically and projectively related to a surface, (3) to introduce and characterize new configurations which are covariantly related to a surface, and (4) to solve both recognized unsolved problems and new problems which present themselves in the theory.

1. Analytic basis. If the homogeneous projective coordinates $y^{(1)}, \cdots, y^{(4)}$ of a general point $y$ on a non-ruled surface $S$ in ordinary space are analytic functions of two independent variables $u, v$, and if the parametric net on $S$ is the asymptotic net, the functions $y^{(i)}$ are solutions of a system of differential equations, which by a suitable transformation can be reduced to Wilczynski's canonical form

$$
y_{u u}+2 b y_{v}+f y=0, \quad y_{v v}+2 a^{\prime} y_{u}+g y=0 .
$$

The coefficients of these equations are functions of $u, v$, which are connected by three conditions of integrability.

In the notation employed by Green [1, p. 86], points $\rho$ and $\sigma$ on the $u$-and $v$-tangents to $S$ at $y$, respectively, are given by

$$
\rho=y_{u}-\beta y, \quad \sigma=y_{v}-\alpha y,
$$

* Presented to the Society, September 6, 1938, under the title Integral invariants of projective differential geometry; $\$ 11$ was presented April 9, 1937, under the title Geometric characterizations in projective differential geometry of curved surfaces; received by the editors May 19, 1939. 
where $\alpha, \beta$ are functions of $u, v$. The line $l$ joining the points $\rho, \sigma$ generates a congruence $\Gamma$ as $y$ varies over $S$.

A line $l^{\prime}$ through a general point $y$ of $S$, but not lying in the tangent plane to $S$ at $y$, joins the point $y$ to the point $z$ given by

$$
z=y_{u v}-\alpha y_{u}-\beta y_{v},
$$

wherein $\alpha, \beta$ are functions of $u, v$. As $y$ varies over $S$ the line $l^{\prime}$ generates a congruence $\Gamma^{\prime}$. In accordance with the classification which Wilczynski introduced with his directrices of the first and second kinds [1, p. 95], we shall say that the line $l$ and congruence $\Gamma$ are of the first kind, and that the line $l^{\prime}$ and the congruence $\Gamma^{\prime}$ are of the second kind.* If the functions $\alpha, \beta$ are the same in equations (1.2), (1.3), the lines $l, l^{\prime}$ are called reciprocal lines because they are reciprocal polar lines with respect to the quadric of Lie at the point $y$. The congruences $\Gamma, \Gamma^{\prime}$, generated by reciprocal lines, are called $r e$ ciprocal congruences.

Throughout this paper, when no statement is made regarding the tetrahedron of reference for local coordinates of points or planes, the tetrahedron will be that whose vertices are $y, y_{u}, y_{v}, y_{u v}$. In this coordinate system, the equations for the line $l$ are

$$
x_{1}+\beta x_{2}+\alpha x_{3}=0, \quad x_{4}=0,
$$

and the equations for $l^{\prime}$ are

$$
x_{2}+\alpha x_{4}=0, \quad x_{8}+\beta x_{4}=0 .
$$

An arbitrary one-parameter family of curves on $S$ is defined by the curvilinear equation

$$
d v-\lambda d u=0,
$$

where $\lambda$ is an arbitrary function of $u, v$. We shall throughout this paper denote by $F_{\lambda}$ the family defined by (1.6), and by $C_{\lambda}$ the curve of the family which passes through the point $y$. The conjugate net $N_{\lambda}$ of which $F_{\lambda}$ is a family is defined by the curvilinear differential equation

$$
d v^{2}-\lambda^{2} d u^{2}=0 .
$$

We denote by $C_{\lambda}$ and $C_{-\lambda}$ the two curves of $N_{\lambda}$ which pass through the point $y$.

2. The $R_{\lambda}$-associate of a line $l$. As a point $y$ of $S$ moves along the curve $C_{\lambda}$, the $u$-tangent at $y$ describes a ruled surface $R_{\lambda}{ }^{(u)}$ and the $v$-tangent at $y$

* Green [1, p. 114] used this means of classifying his canonical edges of the first and second kinds. Fubini and Cech [1, pp. 96-102] have used the same means of classification but have reversed the names. 
describes the ruled surface $R_{\lambda}{ }^{(v)}$. The well known asymptotic ruled surfaces $R^{(u)}$ and $R^{(v)}$ are the special surfaces $R_{\infty}{ }^{(u)}$ and $R_{0}{ }^{(v)}$ in which the curves $C_{\infty}$ and $C_{0}$ are the asymptotic $v$ - and $u$-curves, respectively.

Since the ruled surfaces $R_{\lambda}{ }^{(u)}$ and $R^{(u)}$ have at $y$ the $u$-tangent to $S$ as common generator, the plane which is tangent to $R_{\lambda}{ }^{(u)}$ at a given point $\rho$ of this generator is tangent to $R^{(u)}$ at another point $\rho_{\lambda}$ of the generator. Likewise, since the ruled surfaces $R_{\lambda}{ }^{(v)}$ and $R^{(v)}$ have at $y$ the $v$-tangent to $S$ as common generator, the plane which is tangent to $R_{\lambda}(v)$ at a given point $\sigma$ of this generator is tangent to $R^{(v)}$ at another point $\sigma_{\lambda}$ of the generator. The points $\rho_{\lambda}$ and $\sigma_{\lambda}$ will be called the $R_{\lambda}$-transforms of the points $\rho$ and $\sigma$. respectively. The line $l_{\lambda}$ joining the points $\rho_{\lambda}, \sigma_{\lambda}$ and the congruence $\Gamma_{\lambda}$ generated by $l_{\lambda}$ as $y$ varies over $S$ will be called the $R_{\lambda}$-associates of the line $l$ and congruence $\Gamma$, respectively. The reciprocals of $l_{\lambda}$ and $\Gamma_{\lambda}$ will be called the $R_{\lambda}{ }^{\prime}$ associates of the reciprocals of $l$ and $\Gamma$, respectively.

Let the point $\rho_{\lambda}$ be given by $\rho_{\lambda}=y_{u}-\beta_{\lambda} y$, where $\beta_{\lambda}$ is to be determined by the condition that the point $\left(\rho_{\lambda}\right)_{v}$ shall lie in the plane determined by the points $y, \rho$, and $\rho_{u}+\lambda \rho_{v}$. This condition is satisfied if, and only if, the line $r_{\lambda}$ joining the points $\rho_{u}+\lambda \rho_{v},\left(\rho_{\lambda}\right)_{v}$ cuts the corresponding $u$-tangent of $S$. By making use of equations (1.1) it may be shown that the line $r_{\lambda}$ intersects the tangent plane to $S$ at $y$ in the point whose general coordinates are given by

$$
\rho_{u}+\lambda \rho_{v}-\lambda\left(\rho_{\lambda}\right)_{v}=\lambda\left(\beta_{\lambda}-\beta-2 b / \lambda\right) y_{v}+f_{1} y_{u}+f_{2} y,
$$

where $f_{1}$ and $f_{2}$ are nonzero functions of $u, v$. This point lies on the $u$-tangent if, and only if,

$$
\beta_{\lambda}=\beta+2 b / \lambda .
$$

In a similar manner the expression for the coordinates of $\sigma_{\lambda}$ is found to be

$$
\sigma_{\lambda}=y_{v}-\alpha_{\lambda} y
$$

where $\alpha_{\lambda}=\alpha+2 a^{\prime} \lambda$.

3. The determination of the reference tetrahedra of Green. The general development of Green for the equation of a surface was referred to the tetrahedron whose vertices are points $y, \rho, \sigma$ and $\tau$, where $\tau$ is given by

$$
\tau=y_{u v}-\alpha y_{u}-\beta y_{v}+\alpha \beta y
$$

in which $\alpha, \beta$ are the same functions as those in (1.2) associated with the points $\rho, \sigma$. The point $\tau$ lies on the line $l^{\prime}$ which is the reciprocal of the line $l$ joining $\rho, \sigma$. If the functions $\alpha, \beta$ are chosen suitably, the points $\rho, \sigma$ and $\tau$ become covariant points, the coefficients in the development become absolute invariants, and the development is said to be a canonical development. Since 
geometric determinations for the covariant points $\rho, \sigma$ which are associated with the various canonical developments are well known, the completion of the problem of the determination of the covariant tetrahedra of Green is accomplished by the geometric characterization of the associated points $\tau$.

Green $[1$, p. 98] has shown that the tangents at the points $\rho, \sigma$ to the curved asymptotics of $R^{(u)}, R^{(v)}$, respectively, intersect in the point $\omega$ given by

$$
\omega=\tau-2 a^{\prime} b y,
$$

where $\tau$ corresponds to $\rho, \sigma$. The point $\omega_{\lambda}$ which is similarly defined with reference to the points $\rho_{\lambda}, \sigma_{\lambda}$ is given by $\omega_{\lambda}=\tau_{\lambda}-2 a^{\prime} b y$. By expressing the right member of this equation in terms of $y, \rho, \sigma$, and $\tau$ we have

$$
\omega_{\lambda}=\tau+2 a^{\prime} b y-2 a^{\prime} \lambda \rho-2 b \sigma / \lambda .
$$

If the point $y$ is kept fixed, while the function $\lambda$ is varied, the line joining $y$ and $\omega_{\lambda}$ describes a quadric cone whose equation when referred to $y, \rho, \sigma, \tau$ is found to be

$$
x_{2} x_{3}-4 a^{\prime} b x_{4}^{2}=0 .
$$

Moreover, since fcr every value of $\lambda$ the expression $\omega_{\lambda}$ is a linear combination of $\tau+2 a^{\prime} b y, \rho$ and $\sigma$, the points $\omega_{\lambda}$ all lie in the plane $\pi_{\omega}$ determined by these three points. The locus of the points $\omega_{\lambda}$ as $\lambda$ is varied is therefore a conic. The point $\tau+2 a^{\prime} b y$ is the intersection of the plane $\pi_{\omega}$ with the line $l^{\prime}$. Finally, the point $\tau$ is the harmonic conjugate of $y$ with respect to the points $\tau+2 a^{\prime} b y$ and $\tau-2 a^{\prime} b y$.

We find also that the cone (3.4) intersects the tangent plane in the asymptotic tangents to $S$ at $y$. The planes which are tangent to the cone along the $u$-and v-tangents to $S$ at $y$ intersect in the line $l^{\prime}$ which is the reciprocal of the line joining $\rho, \sigma$.

4. The family of $R_{\lambda}$-derived curves and the curves of Darboux and of Segre. Let $Q_{\lambda}$ denote the point of intersection of a line $l$ with its $R_{\lambda}$-associate $l_{\lambda}$. The point $Q_{\lambda}$ is given by

$$
Q_{\lambda}=a^{\prime} \lambda^{2} \rho-b \sigma .
$$

The direction of the tangent to $S$ at $y$ joining the points $y, Q_{\lambda}$ is given by

$$
d v / d u=-b / a^{\prime} \lambda^{2}
$$

where the right member is evaluated at the point $y$. The tangent line in this direction will be called the $R_{\lambda}$-correspondent of the tangent to the curve $C_{\lambda}$ at $y$. The one-parameter family of curves defined by the curvilinear differential equation

$$
a^{\prime} \lambda^{2} d v+b d u=0
$$


will be called the family of $R_{\lambda}$-derived curves. This family is completely characterized by the property that at a general point $y$ of $S$ the tangent to the $R_{\lambda}$-derived curve through $y$ is the $R_{\lambda}$-correspondent of the tangent to the curve $C_{\lambda}$ which passes through the point. We observe that the family of $R_{\lambda}$-derived curves is independent of the choice of the congruence $\Gamma$ used in

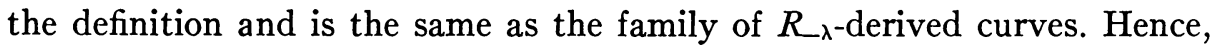
we may associate the $R_{\lambda}$-derived curves with a conjugate net.

Since the curvilinear differential equation for the curves of Darboux is

$$
a^{\prime} d v^{3}+b d u^{3}=0,
$$

and that for the curves of Segre is

$$
a^{\prime} d v^{3}-b d u^{3}=0,
$$

the following theorems are immediate consequences.

THEOREM 4.1. A curve $C_{\lambda}$ is a curve of Darboux if, and only if, at each of its points the $R_{\lambda}$-correspondent of the tangent to $C_{\lambda}$ coincides with this tangent.

TheOREM 4.2. A curve $C_{\lambda}$ is a curve of Segre if, and only if, at each of its points the tangent to $C_{\lambda}$ and its $R_{\lambda}$-correspondent are conjugate tangents of $S$.

5. Pencils of conjugate nets; the Segre-Darboux pencil. The class of $\infty^{1}$ conjugate nets on $S$, every one of which has the property that at every point of the surface its two tangents form with the tangents of a fundamental conjugate net the same cross ratio, is called a pencil of conjugate nets (Wilczynski [2, p. 216]). The differential equation of a general net $N_{h \lambda_{1}}$ of the pencil $p_{\lambda_{1}}$ of conjugate nets determined by the fundamental net $N_{\lambda_{1}}$, defined by $d v^{2}-\lambda_{1}^{2} d u^{2}=0$, is of the form

$$
\left.d v^{2}-h^{2} \lambda_{1}^{2} d u^{2}=0, \quad \text { (h= const. }\right) .
$$

Definition 5.1. The conjugate net $N_{\lambda_{1}}$, where $\lambda_{1}=-b / a^{\prime} \lambda^{2}$ will be called the $R_{\lambda}$-derived conjugate net associated with the family $F_{\lambda}$ defined by (1.6). The associated pencil $p_{\lambda_{1}}$ will be called the $R_{\lambda}$-derived pencil of conjugate nets.

The curves of Darboux and the curves of Segre belong to a pencil of conjugate nets called the Segre-Darboux pencil. The curvilinear differential equation for this pencil is $(5.1)$, where $\lambda_{1}=\left(b / a^{\prime}\right)^{1 / 3}$. As an immediate consequence of the form of this equation we have

THEOREM 5.1. If a conjugate net $N_{\lambda}$ is contained in the associated $R_{\lambda}$-derived pencil of conjugate nets, the pencil is the Segre-Darboux pencil.

The following theorem presents an additional characteristic property of this pencil. 
Theorem 5.2. A conjugate net $N_{\lambda}$ belongs to the Segre-Darboux pencil of conjugate nets if, and only if, at a general point $y$ of $S$, its axis lies in the osculating plane of the $R_{\lambda}$-derived curve at this point.

Let $C_{\bar{\lambda}}$ denote the $R_{\lambda}$-derived curve which passes through the point $y$. The direction of $C_{\bar{\lambda}}$ at $y$ is given by $d v-\bar{\lambda} d u=0$, where $\bar{\lambda}=-b / a^{\prime} \lambda^{2}$. The equation of the osculating plane of $C_{\bar{\lambda}}$ at $y$ is

$$
2 \bar{\lambda}^{2} x_{2}-2 \bar{\lambda} x_{3}+\left(\bar{\lambda}^{\prime}-2 b+2 a^{\prime} \bar{\lambda}^{3}\right) x_{4}=0 .
$$

The axis at $y$ of the conjugate net $N_{\lambda}$ is the line joining the point $y$ and the point $z$ given by (1.3), wherein

$$
\alpha=(\log \lambda)_{v} / 2-b / \lambda^{2}, \quad \beta=-(\log \lambda)_{u} / 2-a^{\prime} \lambda^{2} .
$$

The axis of $N_{\lambda}$ at $y$ lies in the plane (5.2) if the local coordinates $(0,-\alpha,-\beta, 1)$ of the point $z$ satisfy equation (5.2). It is convenient to express this condition in terms of $\bar{\lambda}$. Making use of the following relations,

$$
\begin{aligned}
(\log \lambda)_{v} / 2-b / \lambda^{2} & =\left(\log k b / a^{\prime} \bar{\lambda}\right)_{v} / 4+a^{\prime} \bar{\lambda}, \\
-(\log \lambda)_{u} / 2-a^{\prime} \lambda^{2} & \left.=-\left(\log k b / a^{\prime} \bar{\lambda}\right)_{u} / 4+b / \bar{\lambda}, \quad \text { (k = const. }\right),
\end{aligned}
$$

we obtain this condition in the form

$$
\left.2 \bar{\lambda}^{\prime}=\bar{\lambda}\left(\left[\log k b / a^{\prime} \bar{\lambda}\right]_{u}+\dot{[} \log k b / a^{\prime} \bar{\lambda}\right]_{v} \bar{\lambda}\right),
$$

which may be reduced to the simpler form

$$
2[\log \bar{\lambda}]^{\prime}=\left[\log k b / a^{\prime} \bar{\lambda}\right]^{\prime},
$$

in which accents indicate differentiation with respect to the independent variable $u$, and $d v / d u=\bar{\lambda}$. On integrating (5.3) we obtain

$$
\log \bar{\lambda}^{2}=\log \left(k b / a^{\prime} \bar{\lambda}\right), \quad(k=\text { arb. const. }) .
$$

Hence, we have

$$
\bar{\lambda}=\epsilon\left(k b / a^{\prime}\right)^{1 / 3}, \quad\left(\epsilon^{3}=1\right) .
$$

Solving for $\lambda$, making use of the relation $\bar{\lambda}^{2}=-b / a^{\prime} \lambda^{2}$, we obtain

$$
\lambda= \pm i \epsilon(1 / k)^{1 / 6}\left(b / a^{\prime}\right)^{1 / 3}, \quad\left(i=(-1)^{1 / 2}\right) .
$$

Since $k$ is an arbitrary constant, the net $N_{\lambda}$, where $\lambda$ is given by (5.5), belongs to the Segre-Darboux pencil. This establishes the sufficiency of the condition. The condition can be shown to be necessary by interchanging the hypothesis and conclusion of the sufficiency proof, and reversing the argument. 
The conjugate nets of simplest description, which have the property described in this theorem, are the Segre-Darboux nets. Without the foregoing argument it is clear that the Segre-Darboux nets have the property, since from the theorems of $\$ 5$ we have that the $R_{\lambda_{i}}$-derived family of a SegreDarboux net $N_{\lambda_{i}},(i=1,2,3)$, is the family of Darboux curves of the net.

6 . Projective characterizations for the $\Gamma$-curves of a congruence $\Gamma$. Green defined the $\Gamma$-curves of the congruence $\Gamma$ to be the curves of $S$ which correspond to the developables of the congruence $\Gamma$. New projective characterizations for these curves will be presented in this section.

As $y$ varies over the surface $S$, the points $\rho$ and $\sigma$ of a line of the first kind generate transversal surfaces $S_{\rho}$ and $S_{\sigma}$ of a congruence $\Gamma$. Since corresponding points $y, \rho$ and $\sigma$ have the same curvilinear coordinates $(u, v)$, corresponding directions at these points are defined by the same ratio $d v / d u=\lambda$ and the correspondences between pencils of tangents at $y$, at $\rho$, and at $\sigma$, are projectivities. Let us denote by $\pi_{y}, \pi_{\rho}$ and $\pi_{\sigma}$ the planes which are tangent to $S, S_{\rho}$, and $S_{\sigma}$ at $y, \rho$, and $\sigma$, respectively. For an unspecialized surface $S$ the tangent planes $\pi_{\rho}$ and $\pi_{\sigma}$ intersect in a line $h$, and the two projective pencils of tangents at $\rho$ and $\sigma$ determine a projectivity on $h$ which has two distinct double points $P_{1}$ and $P_{2}$. The two directions $\lambda_{1}, \lambda_{2}$ which correspond to these double points are therefore (for an unspecialized surface) the only ones for which the points $\rho, \rho_{u}+\lambda \rho_{v}, \sigma_{u}+\lambda \sigma_{v}$ are coplanar. The condition that these points be coplanar is necessary and sufficient that for this direction the line $l$ joining $\rho, \sigma$ describes a developable surface of $\Gamma$. Hence, $\lambda_{1}$ and $\lambda_{2}$ are the directions of the $\Gamma$-curves of $S$ at $y$. Moreover, if we consider the definition of the $R_{\lambda}$-associate, it is clear that the reciprocals $l_{\lambda_{1}}, l_{\lambda_{2}}$ of the lines joining $y$ and $P_{1}$, and $y$ and $P_{2}$, respectively, are the $R_{\lambda_{1}}$ and $R_{\lambda_{2}}$-associates of $l$. Hence, the lines joining $y$ and $P_{1}$, and $y$ and $P_{2}$, are the $R_{\lambda_{1}^{\prime}}$ - and $R_{\lambda_{2}^{\prime}}$-associates, respectively, of the line $l^{\prime}$ which is the reciprocal of $l$. We have, therefore, the following theorem:

THEOREM 6.1. If, and only if, at each point $y$ of a curve $C_{\lambda}$ of $S$, the line of the $R_{\lambda}^{\prime}$-associate of the reciprocal congruence $\Gamma^{\prime}$ passes through the line $h$ of intersection of the tangent planes to $S_{\rho}$ at $\rho$ and $S_{\sigma}$ at $\sigma$, the curve is a $\Gamma$-curve of the congruence $\Gamma$.

By considering polar reciprocals with respect to a quadric of Darboux, we obtain

THEOREM 6.2. A curve $C_{\lambda}$ of $S$ is a $\Gamma$-curve of a congruence $\Gamma$ if, and only if, at each point $y$ of the curve the pole of the plane $\pi_{h}$, determined by the point $y$ and the line of intersection of the tangent planes to $S_{\rho}$ at $\rho$ and $S_{\sigma}$ at $\sigma$, with respect to a quadric of Darboux lies on the $R_{\lambda}$-associate of the line of the congruence $\Gamma$ which corresponds to the point $y$. 
The curvilinear differential equation for the net of $\Gamma$-curves of $S$ may be put in the form

$$
2 b\left(\alpha-\alpha_{(a)}\right) d u^{2}+\left(\beta_{v}-\alpha_{u}\right) d v d u-2 a^{\prime}\left(\beta-\beta_{(a)}\right) d v^{2}=0,
$$

wherein $\alpha_{(a)}=-\left(f+\beta_{u}+\beta^{2}\right) / 2 b, \beta_{(a)}=-\left(g+\alpha_{v}+\alpha^{2}\right) / 2 a^{\prime}$. The equations for the planes $\pi_{\rho}$ and $\pi_{\sigma}$ may be shown to be

$$
\begin{aligned}
& x_{1}+\beta x_{2}+\alpha_{(a)} x_{3}+\left(\beta \alpha_{(a)}+\beta_{v}\right) x_{4}=0, \\
& x_{1}+\beta_{(a)} x_{2}+\alpha x_{3}+\left(\alpha \beta_{(a)}+\alpha_{u}\right) x_{4}=0,
\end{aligned}
$$

respectively. By making use of these equations in connection with equation (6.1) for the $\Gamma$-curves, the following theorems may be easily proved.

THEOREM 6.3. The planes $\pi_{\rho}$ and $\pi_{\sigma}$ intersect the line $l^{\prime}$ which is the reciprocal of the line joining $\rho, \sigma$ in one and the same point if, and only if, $\beta_{v}=\alpha_{u}$.

THEOREM 6.4. If the planes $\pi_{\rho}$ and $\pi_{\sigma}$ intersect in a line $h$ which contains a point of $l^{\prime}$, the $\Gamma$-curves form a conjugate net when $h$ passes through neither asymptotic tangent of $S$ at $y$, but they coincide with the family of asymptotic $v$-curves (or u-curves) of $S$ when $h$ passes through the asymptotic u-tangent (or $v$-tangent) to $S$ at $y$.

THEOREM 6.5. If, and only if, the plane $\pi_{\rho}$ coincides with the plane $\pi_{\sigma}$, the $\Gamma$ curves are indeterminate.

THEOREM 6.6. If $\beta_{v} \neq \alpha_{u}$, the planes $\pi_{\rho}$ and $\pi_{\sigma}$ intersect in a line $h$ which is not coplanar with $l^{\prime}$. If the line $h$ contains the point $\rho$ (or $\left.\sigma\right)$, one family of the net of $\Gamma$-curves is the family of asymptotic v-curves (or $u$-curves). If $h$ coincides with the line $l$, the net of $\Gamma$-curves coincides with the asymptotic net on $S$.

Definition 6.1. A congruence which satisfies the hypothesis of Theorem 6.3 will be called central to $S$.

DeFinition 6.2. Let $\sigma_{(a)}$ denote the intersection of the plane $\pi_{\rho}$ with the tangent to the asymptotic v-curve of $S$ at $y$, and let $\rho_{(a)}$ denote the intersection of the plane $\pi_{\sigma}$ with the tangent to the asymptotic u-curve of $S$ at $y$. The line $l_{(a)}$ joining $\rho_{(a)}, \sigma_{(a)}$ and the congruence $\Gamma_{(a)}$ generated by $l_{(a)}$ as $y$ varies over $S$ will be called the asymptotic associates of the line $l$ and congruence $\Gamma$ respectively.

The points $\rho_{(a)}, \sigma_{(a)}^{*}$ are given by

$$
\rho_{(a)}=y_{u}-\beta_{(a)} y, \quad \sigma_{(a)}=y_{v}-\alpha_{(a)} y,
$$

where $\beta_{(a)}, \alpha_{(a)}$ are the functions which appear in (6.1).

* The points $\rho_{(a)}, \sigma_{(a)}$ may also be characterized as follows. The point $\rho_{(a)}$ is the intersection of the $v$-tangent of $S_{\sigma}$ at $\sigma$ with the $u$-tangent of $S$ at $y$, and the point $\sigma_{(a)}$ is the intersection of the $u$-tangent of $S_{\rho}$ at $\rho$ with the $v$-tangent of $S$ at $y$. 
The $\Gamma^{\prime}$-curves are the curves of $S$ which correspond to the developables of the congruence $\Gamma^{\prime}$; they are in fact the intersections of the surface $S$ with the developables of the congruence $\Gamma^{\prime}$.

DEFINITION 6.3. If one of the two families of curves which form a conjugate net consists of $\Gamma^{\prime}$-curves, we shall call the other a family of reflected $\Gamma^{\prime}$-curves.

Green $[1$, p. 93] defined reflected $\Gamma$-curves in a similar manner.

The curvilinear differential equations for the net of $\Gamma^{\prime}$-curves of $S$ may be put in the form

(6.5) $\left(2 b_{v}-2 b\left[\alpha+\alpha_{(a)}\right]\right) d u^{2}+\left(\beta_{v}-\alpha_{u}\right) d u d v+\left(2 a^{\prime}\left[\beta+\beta_{(a)}\right]-2 a_{u}^{\prime}\right) d v^{2}=0$.

The equation for the net of reflected $\Gamma^{\prime}$-curves is

(6.6) $\left[2 b\left(\alpha+\alpha_{(a)}\right)-2 b_{v}\right] d u^{2}+\left(\beta_{v}-\alpha_{u}\right) d u d v+\left[2 a_{u}^{\prime}-2 a^{\prime}\left(\beta+\beta_{(a)}\right)\right] d v^{2}=0$.

The $\Gamma$-curves coincide with the reflected $\Gamma^{\prime}$-curves if, and only if

$$
\alpha_{(a)}=b_{v} / 2 b, \quad \beta_{(a)}=a_{u}^{\prime} / 2 a^{\prime} .
$$

For this selection the line $l_{(a)}$ is the directrix of the first kind of Wilczynski. Hence we have

TheOREM 6.7. The $\Gamma$-curves of a congruence $\Gamma$ coincide with the reflected $\Gamma^{\prime}$ curves of the reciprocal congruence $\Gamma^{\prime}$ if, and only if, the asymptotic associate of the congruence $\Gamma$ is the congruence generated by the directrix of the first kind of Wilczynski.

The following theorem may be proved similarly. Let $P_{(a)}$ denote the point of intersection of the line $l$ with its asymptotic associate $l_{(a)}$. Let $t_{(a)}$ denote the tangent to $S$ at $y$ which passes through $P_{(a)}$, and let $t_{(a)}^{\prime}$ denote the tangent to $S$ at $y$ which is conjugate to $t_{(a)}$ at $y$.

THEOREM 6.8. The $\Gamma^{\prime}$-curves of a congruence $\Gamma^{\prime}$ are indeterminate if, and only if, the following conditions are satisfied: (1) the congruence $\Gamma$ is central to $S$, (2) the pencil of lines whose center is the point $P_{(a)}$ contains the directrix of the first kind of Wilczynski, and (3) the tangent line $t_{(a)}$ and the directrix of the first kind, separate harmonically the lines $l$ and $l_{(a)}$.

The second and third conditions in the theorem may be replaced by the following ones: $\left(2^{\prime}\right)$ the pencil of lines determined by the lines $l^{\prime}$ and $l_{(a)}^{\prime}$ contains the directrix of the second kind of Wilczynski, as well as the tangent $t_{(a)}^{\prime}$ which is conjugate to $t_{(a)},\left(3^{\prime}\right)$ the tangent line $t_{(a)}^{\prime}$ and the directrix of the second kind separate harmonically the lines $l^{\prime}$ and $l_{(a)}^{\prime}$.

7. Theorems on conjugate nets. According to Theorems 6.4 and 6.5 the congruences central to $S$ consist of (1) congruences harmonic to $S$, (2) congruences 
whose $\Gamma$-curves coincide with an asymptotic family of $S$, and (3) congruences whose $\Gamma$-curves are indeterminate.

Definition 7.1. A family $F_{\lambda}$ defined by (1.6) belongs to class $\mathbb{E}$ if the $R_{\lambda}$-associate of a congruence central to $S$ is likewise central to $S$.

The analytic condition that $F_{\lambda}$ belong to class $\mathbb{S}$ is that $\lambda$ satisfy the partial differential equation

$$
(b / \lambda)_{v}-\left(a^{\prime} \lambda\right)_{u}=0 .
$$

If a direction $d v / d u=\lambda$ satisfies equation (7.1), its conjugate direction $d v / d u=-\lambda$ likewise satisfies it. Hence, we have that if a family belongs to class $\mathfrak{E}$, the conjugate net $N_{\lambda}$, of which $F_{\lambda}$ is a family, belongs to class $\mathfrak{E}$.

ThEOREM 7.1. If a family $F_{\lambda}$ of curves of $S$ belongs to class $\mathfrak{\complement}$, the $R_{\lambda}$-derived conjugate net consists of a one-parameter family of projective geodesics and the family of $R_{\lambda}$-derived curves.

The function $\lambda$ satisfies equation (7.1) which may be put in the form

$$
\bar{\lambda}(\log \lambda)_{v}+(\log \lambda)_{u}=-\left(\log a^{\prime}\right)_{u}+\bar{\lambda}(\log b)_{v},
$$

wherein $\bar{\lambda}=b / a^{\prime} \lambda^{2}$. We obtain from the equation $\lambda^{2}=b / a^{\prime} \bar{\lambda}$ by logarithmic differentiation, the relations

$$
\begin{aligned}
& (\log \lambda)_{v}=\left[\left(\log b / a^{\prime}\right)_{v}-(\log \bar{\lambda})_{v}\right] / 2, \\
& (\log \lambda)_{u}=\left[\left(\log b / a^{\prime}\right)_{u}-(\log \bar{\lambda})_{u}\right] / 2 .
\end{aligned}
$$

Using these forms, we may express equation (7.2) entirely in terms of $\bar{\lambda}$. On simplifyng the resulting equation we obtain

$$
\bar{\lambda}_{u}+\bar{\lambda} \bar{\lambda}_{v}=\left(\log a^{\prime} b\right)_{u} \bar{\lambda}-\left(\log a^{\prime} b\right)_{v} \bar{\lambda}^{2} .
$$

Putting $\bar{\lambda}=d v / d u$ and $\bar{\lambda}_{u}+\bar{\lambda} \bar{\lambda}_{v}=d^{2} v / d u^{2}$, we have the usual form of the differential equation for the projective geodesics

$$
d^{2} v / d u^{2}=\left(\log a^{\prime} b\right)_{u} d v / d u-\left(\log a^{\prime} b\right)_{v}(d v / d u)^{2} .
$$

Hence the curves defined by $d v-\bar{\lambda} d u=0$, where $\bar{\lambda}=b / a^{\prime} \lambda^{2}$, are projective geodesics under the hypothesis of the theorem. Since, moreover, the family of $R_{\lambda}$-derived curves is defined by $d v-\lambda_{1} d u=0$, where $\lambda_{1}=-b / a^{\prime} \lambda^{2}$, the families $F_{\bar{\lambda}}$ and $F_{\lambda_{1}}$ form the $R_{\lambda}$-derived conjugate net.

THEOREM 7.2. If a one-parameter family of projective geodesics and the family of $R_{\lambda}$-derived curves associated with a family $F_{\lambda}$ form a conjugate net, the family $F_{\lambda}$ belongs to class $\mathfrak{S}$. 
A one-parameter family of projective geodesics is defined by $d v-\bar{\lambda} d u=0$, where $\bar{\lambda}$ is a solution of the equation (7.5). According to hypothesis, $\bar{\lambda}=b / a^{\prime} \lambda^{2}$. Hence, we have

$$
\bar{\lambda}_{u}+\bar{\lambda} \bar{\lambda}_{v}=\left(b / a^{\prime} \lambda^{2}\right)_{u}+\left(b / a^{\prime} \lambda^{2}\right)\left(b / a^{\prime} \lambda^{2}\right)_{v} .
$$

Equating the right members of (7.4) and (7.6) and simplifying, we obtain

$$
\left(\log a^{\prime} \lambda\right)_{u}=\left(b / a^{\prime} \lambda^{2}\right)(\log b / \lambda)_{v},
$$

which is equivalent to equation (7.1).

The functions $\alpha, \beta$ for the axis congruence and the associate axis congruence of the net $N_{\lambda}$ are given by

$$
\begin{array}{ll}
\alpha=(\log \lambda)_{v} / 2-b / \lambda^{2}, & \beta=-(\log \lambda)_{u} / 2-a^{\prime} \lambda^{2}, \\
\alpha=(\log \lambda)_{v} / 2+b / \lambda^{2}, & \beta=-(\log \lambda)_{u} / 2+a^{\prime} \lambda^{2},
\end{array}
$$

respectively. Hence, we have

THEOREM 7.3. If $F^{-}$denotes the family of $R_{\lambda}$-derived curves associated with a conjugate net $N_{\lambda}$, the axis congruence of the net is the $R_{\bar{\lambda}}{ }^{\prime}$-associate of the associate axis congruence of the net.

Since, moreover, the ray congruence of a conjugate net is the reciprocal of the associate axis congruence of the net, and the associate ray congruence is the reciprocal of the axis congruence, we have

THEOREM 7.4. If $F_{\bar{\lambda}}$ denotes the family of $R_{\lambda}$-derived curves associated with a conjugate net $N_{\lambda}$, the associate ray congruence of the net is the $R_{\bar{\lambda}}$-associate of the ray congruence of the net.

8. The $R_{\lambda, j, k}$-associates of a line $l$ and congruence $\Gamma$; the transformations of Čech. The concept of the $R_{\lambda}$-associate of a line $l$ may be generalized as follows. Let $\rho_{\lambda, j}$ denote the point on the asymptotic $u$-tangent to $S$ at $y$ which is determined by the cross ratio equation

$$
\left(y, \rho, \rho_{\lambda}, \rho_{\lambda, j}\right)=j, \quad \quad(j=\text { const. }) .
$$

Let $\sigma_{\lambda, k}$ denote the point determined on the $v$-tangent to $S$ at $y$ by the cross ratio equation

$$
\left.\left(y, \sigma, \sigma_{\lambda}, \sigma_{\lambda, k}\right)=k, \quad \text { ( } k=\text { const. }\right) .
$$

The points $\rho_{\lambda, j}$ and $\sigma_{\lambda, k}$ will be called the $R_{\lambda, j^{-}}$and $R_{\lambda, k^{-}}$transforms of $\rho$ and $\sigma$, respectively. The line $l_{\lambda, j, k}$ joining the points $\rho_{\lambda, j}$ and $\sigma_{\lambda, k}$ and the congruence $\Gamma_{\lambda, j, k}$ generated by $l_{\lambda, j, k}$ as $y$ moves over $S$ will be called the $R_{\lambda, j, k}$-associates of $l$ and $\Gamma$ respectively. The reciprocals $l_{\lambda, j, k}^{\prime}$ and $\Gamma_{\lambda, j, k}^{\prime}$ of $l_{\lambda, j, k}$ and $\Gamma_{\lambda, j, k}$ will be called the $R_{\lambda, j, k}^{\prime}$-associates of $l^{\prime}$ and $\Gamma^{\prime}$, respectively. 
The points $\rho_{\lambda, j}$ and $\sigma_{\lambda, k}$ are given by

$$
\rho_{\lambda, j}=\rho-2 j b y / \lambda, \quad \sigma_{\lambda, k}=\sigma-2 k a^{\prime} \lambda y .
$$

The general transformation $\Sigma_{j, k}$ of $\check{C} e c h^{*}[1$, p. 192] is defined analytically by the equations

$$
\begin{aligned}
\xi_{1} & =0, \quad r \xi_{2}=x_{2} x_{3}{ }^{2}, \quad r \xi_{3}=x_{2}^{2} x_{3}, \\
r \xi_{4} & =-x_{1} x_{2} x_{3}-2 b j x_{2}{ }^{3}-2 a^{\prime} k x_{3}{ }^{3}, \quad(j=\text { const. }, k=\text { const. }),
\end{aligned}
$$

where $r$ is a proportionality factor. It is a transformation between points with local coordinates $x$ in the tangent plane of a surface at a point and planes with local coordinates $\xi$ through the point.

We present a new geometric characterization of the general transformation of Cech. Let $t_{\lambda}$ and $t_{-\lambda}$ denote the conjugate tangents to $S$ at $y$ whose directions are $d v / d u=\lambda$, and $d v / d u=-\lambda$, respectively. Let $P_{-\lambda}$ denote the point of intersection of $t_{-\lambda}$ with an arbitrary line $l$ of the first kind.

THEOREM 8.1. The plane $\pi_{\lambda, j, k}$ which corresponds to a point $P_{-\lambda}$ in the transformation $\Sigma_{j, k}$ of $\bar{C}$ ech is the plane which is determined by the tangent $t_{\lambda}$ and the reciprocal of the $R_{\lambda, j, k}$-associate of $l$.

The equations for $t_{-\lambda}$ are $x_{3}+\lambda x_{2}=0, x_{4}=0$. Equations (1.4) are for an arbitrary line $l$. Hence the local coordinates of $P_{-\lambda}$ may be found to be

$$
x_{1}=-\beta+\alpha \lambda, \quad x_{2}=1, \quad x_{3}=-\lambda, \quad x_{4}=0 .
$$

There is a point $Q_{\lambda}$ of $t_{\lambda}$ whose local coordinates are given by $(0,1, \lambda, 0)$, and there is a point $z_{\lambda, j, k}$ of $l_{\lambda, j, k}^{\prime}$ whose local coordinates are $\left(0,-\alpha-2 k a^{\prime} \lambda\right.$, $-\beta-2 j b / \lambda, 1)$. Since the plane determined by $t_{\lambda}$ and $l_{\lambda, j, k}^{\prime}$ contains the points $y, Q_{\lambda}$, and $z_{\lambda, j, k}$, its equation may be found to be

$$
\lambda x_{2}-x_{3}+\left(\alpha \lambda+2 k a^{\prime} \lambda^{2}-\beta-2 j b / \lambda\right) x_{4}=0 .
$$

By substituting the values for $x_{1}, x_{2}, x_{3}, x_{4}$ given by (8.5) in (8.4), we find that the coordinates of the plane $\pi_{\lambda, j, k}$ are the same as those of the plane (8.6), except for a proportionality factor. Hence the theorem is proved.

The correspondence of $C$. Segre is the transformation $\Sigma_{1,1}$. It was defined to be the correspondence between the osculating planes at a point $y$ of $S$ of all of the curves of $S$ passing through $y$ and the corresponding ray-points of these curves at $y$. The geometrical characterization which we have given for $\Sigma_{i, k}$ reduces to a very simple form for $\Sigma_{1,1}$, namely, the plane $\pi_{\lambda}$ which is in the correspondence of Segre with the point $P_{-\lambda}$ is determined by the tangent $t_{\lambda}$ and the line $l_{\lambda}^{\prime}$ which is the reciprocal of the $R_{\lambda}$-associate of $l$.

* Lane [1, p. 209] has characterized geometrically the transformations $\Sigma_{j . k}$, where $j=k$. 
To locate the ray-point of the curve $C_{\lambda}$ at $y$, select in the osculating plane $\pi_{\lambda}$, of the curve $C_{\lambda}$ at $y$, an arbitrary line $l^{\prime}$ of the second kind. The $R_{-\lambda}$-associate of the reciprocal of $l^{\prime}$ intersects the tangent line $t_{-\lambda}$ in the point $P_{-\lambda}$ which is the ray-point of $C_{\lambda}$ at $y$.

The equations in plane coordinates $\xi$ of the pencil of planes whose axis is an arbitrary line $l^{\prime}$ of the second kind are $\xi_{1}=0, \xi_{4}-\alpha \xi_{2}-\beta \xi_{3}=0$, wherein $\alpha, \beta$ are arbitrary functions of $u$, v. It is well known that the points which correspond to these planes in the transformation $\Sigma_{j, k}$ determine a plane cubic in the tangent plane to $S$ at $y$ whose equations are

$$
x_{1} x_{2} x_{3}+\alpha x_{2} x_{3}^{2}+\beta x_{2}^{2} x_{3}+2 j b x_{2}^{3}+2 k a^{\prime} x_{3}^{3}=0, \quad x_{4}=0 .
$$

Let $K_{\lambda, j, k}$ denote the point of intersection of the tangent $t_{\lambda}$ with the $R_{\lambda, j, k^{-}}$ associate of $l$.

THEOREM 8.2. The locus of the point $K_{\lambda, j, k}$ as the direction $\lambda$ is varied at $y$ is the cubic (8.7).

The equations for $t_{\lambda}$ and for $l_{\lambda, j, k}$ are $x_{3}=\lambda x_{2}$ and $x_{1}+(\beta+2 j b / \lambda) x_{2}$ $+\left(\alpha+2 k a^{\prime} \lambda\right) x_{3}=0$, respectively. The point $K_{\lambda, j, k}$ of intersection of these two lines has, therefore, coordinates that are proportional to

$$
x_{1}=-\left(\beta+2 j b / \lambda+\alpha \lambda+2 k a^{\prime} \lambda^{2}\right), \quad x_{2}=1, \quad x_{3}=\lambda .
$$

Homogeneous elimination of $\lambda$ among equations (8.8) gives the equation (8.7) for the cubic.

Among the important cubics (8.7) which have been studied is the one introduced by B. Segre in which all of the $\infty^{4}$ non-composite cubic surfaces having fourth order contact with the surface at the point $y$ cut the tangent plane of the surface at $y$. This cubic is characterized geometrically by Theorem 8.2, wherein $j=k=1 / 3$ and $l$ is the canonical edge of the first kind. Its equations in the notation of this paper are equations (8.7), wherein $j=k=1 / 3$ and $\alpha=a_{v}^{\prime} / 4 a^{\prime}, \beta=b_{u} / 4 b$.

9. Differential invariants. The differential form

$$
d \phi_{j, k}=-2\left(j b d u^{3}+k a^{\prime} d v^{3}\right) / d u d v,
$$$$
(j, k=\text { consts. }) \text {, }
$$

is an absolute invariant under the most general transformation of independent and dependent variables maintaining the asymptotic net as parametric.

To provide a geometric interpretation for $d \phi_{j, k}$ let $Q$ denote a point on the tangent to a curve $C_{\lambda}$ at $y$ and let $K$ and $K_{\lambda, j, k}$ denote the points in which this tangent intersects a line $l$ and its $R_{\lambda, j, k}$ associate line, respectively. We define the $(j, k)$ non-euclidean distance from $y$ to $Q$ to be

$$
D_{y Q}^{(j, k)}=\left(y, K, Q, K_{\lambda, j, k}\right) .
$$


Let $Y$ denote a point near to $y$ on the curve $C_{\lambda}$, and let the curvilinear coordinates of $y$ and $Y$ be $(u, v)$ and $(u+\delta u, v+\delta v)$ respectively, where $\left(\delta u^{2}+\delta v^{2}\right)^{1 / 2} \leqq \epsilon$. Since $Y=y(u+\delta u, v+\delta v)$ and the limit of $\delta v / \delta u$ as $\delta u$ tends to zero as $\lambda(u, v)$, the general coordinates of $Y$ may be given by the expansion

$$
Y=y+\left(y_{u}+\lambda y_{v}\right) \delta u+\text { terms of order }(\delta u)^{2}
$$

wherein $y=y(u, v)$. Hence the coordinates of $Y$ differ only by terms of order $(\delta u)^{2}$ from the point $Y_{1}$ on the tangent to $C_{\lambda}$ at $y$ given by $Y_{1}=y+\left(y_{u}+\lambda y_{v}\right) \delta u$. Therefore the principal parts of the infinitesimal cross ratios

$$
\left(y, K, Y, K_{\lambda, j, k}\right), \quad\left(y, K, Y_{1}, K_{\lambda, j, k}\right)
$$

are identical. It may be easily shown that this principal part is the absolute differential invariant $d \phi_{j, k}$ which we wished to characterize.

It may be observed that $d \phi_{1,1}$ is the projective linear element and $d \phi_{0,1}$ and $d \phi_{1,0}$ are the elementary forms of Bompiani.

The integral of the form $d \phi_{j, k}$ extended over a finite $\operatorname{arc} C_{\lambda}$ is intrinsically and projectively related to this arc. To interpret this integral geometrically let $A$ and $B$ denote the end points of the arc, and let $\left(u_{0}, v_{0}\right)$ and $(u, v)$ denote the curvilinear coordinates of $A$ and $B$, respectively. Let $\epsilon$ be a positive number, and divide the $\operatorname{arc} C_{\lambda}$ by means of the intermediate points $Y_{i}$, $(i=1,2, \cdots, n-1)$, into $n$ smaller arcs. Let the curvilinear coordinates of $Y_{p}$ be $\left(u_{p}, v_{p}\right)$, where $u_{n}=u$ and $v_{n}=v$, and where $\left[\left(u_{p}-u_{p-1}\right)^{2}+\left(v_{p}-v_{p-1}\right)^{2}\right]^{1 / 2} \leqq \epsilon$, $(p=1,2, \cdots, n-1)$, with $\epsilon$ tending to zero as $n$ increases without limit. Then if we put $u_{p}-u_{p-1}=\delta u_{p}$ and $v_{p}-v_{p-1}=\delta v_{p}$, we have

$$
I_{j, k}=\int_{C_{\lambda}} d \phi_{i, k}=\lim _{n \rightarrow \infty} \sum_{p=1}^{n-1} D_{Y_{p-1} Y_{p}}^{(j, k)} .
$$

We have therefore

THEOREM 9.1. The integral $I_{j, k}$ is the limit of the sum of infinitesimal noneuclidean distances, each of which is defined at a separate point $Y_{p-1}$ of $C_{\lambda}$ as the principal part of the corresponding cross ratio $\left(Y_{p-1}, K, Y_{p}, K_{\lambda, j, k}\right)$ which is geometrically determined at $Y_{p-1}$.

This geometric characterization adds to the significance of the extremals of the special integrals which have been studied heretofore. Among these are the pangeodesics which are the extremals of the integral $I_{1,1}$, and the two families of hypergeodesics which are the extremals of the integrals $I_{1,0}$ and $I_{0,1}$, respectively.

The element of projective arc length

$$
d s=2\left(a^{\prime} b d u d v\right)^{1 / 2}
$$


may be characterized geometrically in a somewhat similar manner. The line $l_{\lambda}$ which is the $R_{\lambda}$-associate of $l$, envelops a conic $C$ as the direction $d v / d u=\lambda$ is varied while $(u, v)$ are held constant. The conic passes through the points $\rho$ and $\sigma$ in which $l$ intersects the $u$ and $v$ tangents to $S$ at $y$ and is in fact tangent to these asymptotic tangents at these points. The equation of the conic $C$ when referred to the triangle of reference, whose vertices are the points $y, \rho$, and $\sigma$ may easily be found to be

$$
x_{1}^{2}=16 a^{\prime} b x_{2} x_{3} .
$$

Let $Q_{1}, Q_{2}$ denote the points of intersection of the tangent line to $C_{\lambda}$ at $y$ with the conic (9.4). If we replace, throughout the theory for the characterization of $d \phi_{j, k}$ and $I_{j, k}$, the points $K$ and $K_{\lambda, j, k}$ by the points $Q_{1}$ and $Q_{2}$, we obtain geometric determinations for the form $d s=2\left(a^{\prime} b v^{\prime}\right)^{1 / 2} d u$ and the corresponding integral $I=\int_{c_{\lambda}} 2\left(b a^{\prime} v^{\prime}\right)^{1 / 2} d u$ known as the projective arc-length.

Another interesting invariant, which has been characterized by Bompiani, is $-b / a^{\prime} \lambda^{3}=-d \phi_{1,0} / d \phi_{0,1}$. We find that this invariant may be characterized geometrically by the cross ratio

$$
\left(0, \infty,-b d u^{2} / a^{\prime} d v^{2}, d v / d u\right)
$$

which the tangent line $t_{\lambda}$ makes with the $u$ and $v$ asymptotic tangents and the $R_{\lambda}$-correspondent of $t_{\lambda}$ at the point $y$ of $S$.

10. Pangeodesics and union curves.* The equation of a general quadric which has contact of the second order with a surface $S$ at a point $y$ is

$$
x_{2} x_{3}+x_{4}\left(-x_{1}+k_{2} x_{2}+k_{3} x_{3}+k_{4} x_{4}\right)=0,
$$

where the coefficients $k_{2}, k_{3}, k_{4}$ are arbitrary constants for the fixed point $y$ and functions of $u, v$ when $y$ is varied over $S$. This quadric cuts the surface $S$ in a curve with a triple point at $y$, whose tangents are in the directions satisfying the equation

$$
2 b d u^{3}-3 k_{2} d u^{2} d v-3 k_{3} d u d v^{2}+2 a^{\prime} d v^{3}=0 .
$$

If two of these triple-point tangents coincide in the direction $d v / d u=\lambda$, the third tangent must be in the direction $d v / d u=-b / a^{\prime} \lambda^{2}$. Hence we have the following

THEOREM 10.1. If a quadric having second order contact with $S$ at $y$ intersects $S$ in a curve having two coincident triple-point tangents $t_{\lambda}$ at $y$, in the direction $d v / d u=\lambda$, where $\lambda$ is an arbitrary function of $u$, $v$, the remaining triplepoint tangent is the $R_{\lambda}$-correspondent of $t_{\lambda}$.

* Union curves were introduced by Miss P. Sperry [1, p. 214]. 
For each selection of $\lambda$ there is a pencil of quadrics characterized by the hypothesis of the above theorem. The equation for a general one of these quadrics is given by (9.1) where

$$
k_{2}=\left(4 b / \lambda-2 a^{\prime} \lambda^{2}\right) / 3, \quad k_{3}=\left(4 a^{\prime} \lambda-2 b / \lambda^{2}\right) / 3, \quad\left(k_{4} \text { arbitrary }\right) .
$$

Since the quadric of Moutard at $y$ and in the direction $d v / d u=\lambda$ is one of the quadrics of this pencil, we shall call this pencil the Moutard pencil of quadrics corresponding to the tangent $t_{\lambda}$ at $y$. The following theorem, the proof of which will be left to the reader, characterizes a new line of the first kind in association with an arbitrary line of the second kind.

THEOREM 10.2. The polar reciprocal of an arbitrary line $l^{\prime}$ of the second kind with respect to a quadric of the Moutard pencil corresponding to a tangent $t_{\lambda}$ at $y$ is a line $l_{m, \lambda}$ of the first kind which is dependent on the choice of $l^{\prime}$ and $\lambda$ but is independent of the choice of the quadric of the pencil.

The equations of the line $l_{m, \lambda}$ are easily found to be $(10.4) x_{1}+\left(\beta+\left[2 a^{\prime} \lambda^{2}-4 b / \lambda\right] / 3\right) x_{2}+\left(\alpha+\left[2 b / \lambda^{2}-4 a^{\prime} \lambda\right] / 3\right) x_{3}=0, x_{4}=0$ where $\alpha$ and $\beta$ are the functions associated with the point $z$ on $l^{\prime}$ given by (1.3).

We shall call the line $l_{m, \lambda}$ the $M^{(\lambda)}$-associate of the line $l^{\prime}$, and the congruence $\Gamma_{m, \lambda}$, generated by $l_{m, \lambda}$ as $y$ moves over $S$, the $M^{(\lambda)}$-associate of the congruence $\Gamma^{\prime}$.

Definition 10.1 A family $F_{\lambda}$, defined by (1.6) belongs to class $\mathfrak{S}_{m}$ if the $M^{(\lambda)}$-associate of the reciprocal of a congruence central to $S$ is itself central to $S$.

The analytic condition that $F_{\lambda}$ belong to class $\mathfrak{C}_{m}$ is, therefore, that $\lambda$ satisfy the partial differential equation

$$
\left(a^{\prime} \lambda^{2}-2 b / \lambda\right)_{v}=\left(b / \lambda^{2}-2 a^{\prime} \lambda\right)_{u} .
$$

This is, moreover, the equation for the pangeodesics. Hence we have

Theorem 10.3. A curve is a pangeodesic if, and only if, it belongs to class $\mathfrak{E}_{m}$.

Let us now obtain 'he second order differential equation for the curves $C_{\lambda}$ which are characterized by the property that the $M^{(\lambda)}$-associate of an arbitrarily chosen congruence $\Gamma^{\prime}$ of the second kind is the ray congruence of the associated conjugate net $N_{\lambda}$. Since the functions $\alpha$ and $\beta$ associated with the ray-congruence of a conjugate net $N_{\lambda}$ are given by

$$
\alpha=\left([\log \lambda]_{v}+2 b / \lambda^{2}\right) / 2, \quad \beta=\left(-[\log \lambda]_{u}+2 a^{\prime} \lambda^{2}\right) / 2,
$$


the conditions of the problem require that $\lambda(u, v)$ satisfy the equations

$$
\begin{aligned}
\beta+\left(2 a^{\prime} \lambda^{3}-4 b\right) / 3 \lambda & =\left(-[\log \lambda]_{u}+2 a^{\prime} \lambda^{2}\right) / 2, \\
\alpha+\left(2 b-4 a^{\prime} \lambda^{3}\right) / 3 \lambda^{2} & =\left([\log \lambda]_{v}+2 b / \lambda^{2}\right) / 2 .
\end{aligned}
$$

If we multiply the first of these equations by $-2 \lambda$ and the second by $2 \lambda^{2}$, and add corresponding sides of the resulting equations, we obtain

$$
\lambda_{u}+\lambda \lambda_{v}=2 b-2 \beta \lambda+2 \alpha \lambda^{2}-2 a^{\prime} \lambda^{3} .
$$

If we replace $\lambda$ by $d v / d u$ and $\lambda_{u}+\lambda \lambda_{v}$ by $d^{2} v / d u^{2}$, we obtain

$$
d^{2} v / d u^{2}=2 b-2 \beta d v / d u+2 \alpha(d v / d u)^{2}-2 a^{\prime}(d v / d u)^{3},
$$

which is the well known differential equation for the union curves of the congruence $\Gamma^{\prime}$. Hence, we have

THEOREM 10.4. The curves defined by (1.6), which possess the property that the $M^{(\lambda)}$-associate of a congruence $\Gamma^{\prime}$ of the second kind is the ray congruence of the associated conjugate net $N_{\lambda}$, are union curves of the congruences $\Gamma^{\prime}$.

11. The projective normal. The purpose of this section is to present a new geometric characterization of the projective normal. Consider the points $\tau$ and $\omega$, given by (3.1) and (3.2), respectively, which are the points distinct from $y$ in which an arbitrary line $l^{\prime}$ of the second kind intersects the quadrics of Wilczynski and Lie at $y$. As the point $y$ moves along a curve $C_{\lambda}$, the points $\tau$ and $\omega$ describe corresponding curves. The tangent lines at $\tau$ and $\omega$ to these curves intersect the tangent plane to $S$ at $y$ in points which we denote by $T_{\lambda}$ and $W_{\lambda}$, respectively. The expression for the general coordinates of $T_{\lambda}$ is given by a linear combination of $\tau$ and $\tau_{u}+\lambda \tau_{v}$ which does not contain $y_{u v}$. A similar combination of $\omega$ and $\omega_{u}+\lambda \omega_{v}$ gives the expression for the general coordinates of $W_{\lambda}$. The term of $\tau_{u}+\lambda \tau_{v}$ which involves $y_{u v}$ is $-(\beta+\alpha \lambda) y_{u v}$. The same term appears in $\omega_{u}+\lambda \omega_{v}$. Hence, the expressions for $T_{\lambda}$ and $W_{\lambda}$ are

$$
T_{\lambda}=\tau_{u}+\lambda \tau_{v}+(\beta+\alpha \lambda) \tau, \quad W_{\lambda}=\omega_{u}+\lambda \omega_{v}+(\beta+\alpha \lambda) \omega .
$$

Making use of the expressions for $\tau$ and $\omega$ and the equations (1.1), we obtain

$$
W_{\lambda}-T_{\lambda}=-2 a^{\prime} b\left[y_{u}-\bar{\beta} y+\lambda\left(y_{v}-\bar{\alpha} y\right)\right],
$$

where $\bar{\beta}=-\beta-\left(\log a^{\prime} b\right)_{u}, \bar{\alpha}=-\alpha-\left(\log a^{\prime} b\right)_{v}$. Let $t_{\lambda}$ denote the tangent to $C_{\lambda}$ at $y$, let $r$ denote the line joining $W_{\lambda}$ and $T_{\lambda}$, and let $\nu_{\lambda}$ denote the point of intersection of $t_{\lambda}$ and $r$. Since the right member of (11.1) is a linear combination of the expressions for the coordinates of $W_{\lambda}$ and $T_{\lambda}$, it is the expression for the coordinates of $\nu_{\lambda}$. We shall call the point $\nu_{\lambda}$ the $\nu$-point of $t_{\lambda}$, associated with the line $l^{\prime}$. 
Since the right member of (11.1) is a linear combination of $y_{u}-\bar{\beta} y$ and $y_{v}-\bar{\alpha} y$, the point $\nu_{\lambda}$, for any value of $\lambda$, lies on a straight line which joins $\bar{\rho}$ and $\bar{\sigma}$ given by $\bar{\rho}=y_{u}-\bar{\beta} y, \bar{\sigma}=y_{v}-\bar{\alpha} y$, where $\bar{\beta}$ and $\bar{\alpha}$ are defined above.

We shall call the line $\bar{l}$ the $\nu$-associate of the line $l^{\prime}$, corresponding to the point $y$ of $S$. We state now

THEOREM 11.1. As the direction $\lambda$ is varied at $y$, the $\nu$-point of $t_{\lambda}$, associated with the line $l^{\prime}$, describes a straight line which we call the $\nu$-associate of the line $l^{\prime}$.

The point $\mu$ of intersection of the reciprocal $l$ of $l^{\prime}$ with the line $\bar{l}$ has general coordinates of the form

$$
\mu=(\bar{\alpha}-\alpha)\left(y_{u}+\left[\log a^{\prime} b\right]_{u} y / 2\right)-(\bar{\beta}-\beta)\left(y_{v}+\left[\log a^{\prime} b\right]_{v} y / 2\right) .
$$

Since the intersections of the reciprocal $l_{n}$ of the projective normal with the $u$ and $v$ tangents to $S$ at $y$ are given by

$$
\rho=y_{u}+\left[\log a^{\prime} b\right]_{u} y / 2, \quad \sigma=y_{v}+\left[\log a^{\prime} b\right]_{v} y / 2,
$$

and since $\mu$ is a linear combination of these expressions, we have

THEOREM 11.2. The point $\mu$, which is the intersection of the reciprocal of an arbitrarily chosen line $l^{\prime}$ of the second kind with the $\nu$-associate of $l^{\prime}$, lies on the reciprocal of the projective normal.

If we consider a pair of lines $l_{1}^{\prime}, l_{2}^{\prime}$ of the second kind, we may determine the reciprocal $l_{n}$ of the projective normal as the line joining the points $\mu_{1}$ and $\mu_{2}$ which correspond to $l_{1}^{\prime}$ and $l_{2}^{\prime}$, respectively, at the point $y$.

Let the tangent line to $S$ at $y$, which contains the point $\mu$ in correspondence with $l^{\prime}$ at $y$, be denoted by $t$. The equations for $t$ are

$$
(\bar{\alpha}-\alpha) x_{3}+(\bar{\beta}-\beta) x_{2}=0, \quad x_{4}=0 .
$$

The equations for the lines $l$ and $\bar{l}$ are (1.4) and

$$
x_{1}-\left(\beta+\left[\log a^{\prime} b\right]_{u}\right) x_{2}-\left(\alpha+\left[\log a^{\prime} b\right]_{v}\right) x_{3}=0, \quad x_{4}=0,
$$

respectively. The harmonic conjugate of $t$ with respect to $l$ and $\bar{l}$ is the line $l_{n}$ whose equations are

$$
2 x_{1}-\left(\log a^{\prime} b\right)_{u} x_{2}-\left(\log a^{\prime} b\right)_{v} x_{3}=0, \quad x_{4}=0 .
$$

Hence we have

THEOREM 11.3. The harmonic conjugate of $t$ with respect to the lines $l$ and $\bar{l}$ is the line $l_{n}$ which is the reciprocal of the projective normal.

Let $t^{\prime}$ denote the tangent to $S$ at $y$ which is the conjugate of $t$. Now since $t^{\prime}$ and the lines $l^{\prime}, \bar{l}^{\prime}$, and $l_{n}^{\prime}$ which are reciprocals of $l, \bar{l}$, and $l_{n}$, respectively, are 
also reciprocal polar lines of $t, l, \bar{l}$ and $l_{n}$ with respect to any quadric of Darboux, we have

TheOREM 11.4. The lines $t^{\prime}, l^{\prime}, \bar{l}^{\prime}$ and $l_{n}^{\prime}$ are coplanar, and the harmonic conjugate of $t^{\prime}$ with respect to the lines $l^{\prime}$ and $\bar{l}^{\prime}$ is the projective normal.

12. Hypergeodesics associated with the projective normal. The tangent line $t$ which we have defined in association with an arbitrary line $l^{\prime}$ of the second kind can be used effectively to obtain new geometric characterizations for several important families of hypergeodesics associated with a surface $S$, namely, the projective geodesics, the union curves of the projective normal, and the dual union curves of the projective normal. These characterizations are completely described in the following theorems.

THEOREM 12.1. The tangent $t$ associated with the line $l^{\prime}$ which is the cuspaxis at $y$ of a pencil $p_{\lambda}$, of conjugate nets, and the tangent to the curve $C_{\lambda}$ of the fundamental net $N_{\lambda}$ at $y$ are conjugate tangents if, and only if, the curve is a projective geodesic.

According to the hypothesis we must have

$$
\lambda=\left(2 \beta+\left[\log a^{\prime} b\right]_{u}\right) /\left(2 \alpha+\left[\log a^{\prime} b\right]_{v}\right),
$$

where $\beta=-(\log \lambda)_{u} / 2$ and $\alpha=(\log \lambda)_{v} / 2$. Hence, on clearing of fractions we obtain

$$
\lambda_{u}+\lambda \lambda_{v}=-\left(\log a^{\prime} b\right)_{v} \lambda^{2}+\left(\log a^{\prime} b\right)_{u} \lambda,
$$

which is the equation for the projective geodesics. The substitutions are reversible and therefore the condition is necessary and sufficient.

Theorem 12.2. The tangent $t$ associated with the line $l^{\prime}$ which is the axis of $y$ with respect to a conjugate net $N_{\lambda}$, and the tangent to the curve $C_{\lambda}$ at $y_{2}$ are conjugate tangents if, and only if, the curve is a union curve of the projective normal.

The hypothesis here requires that

where

$$
\lambda=\left[2 \beta+\left(\log a^{\prime} b\right)_{u}\right] /\left[2 \alpha+\left(\log a^{\prime} b\right)_{v}\right],
$$

$$
\beta=\left[-(\log \lambda)_{u}-2 a^{\prime} \lambda^{2}\right] / 2, \quad \alpha=\left[(\log \lambda)_{v}-2 b / \lambda^{2}\right] / 2 .
$$

On clearing of fractions we obtain the equation for the union curves of the projective normal

$$
\lambda_{u}+\lambda \lambda_{v}=2 b+\left(\log a^{\prime} b\right)_{u} \lambda-\left(\log a^{\prime} b\right)_{v} \lambda^{2}-2 a^{\prime} \lambda^{3} .
$$

The argument is again reversible. 
The remaining theorem can be stated by replacing "the axis of $y$ " by "the associate axis of $y$," and "union curve" by "dual union curve." The method of proof is similar to that of the above theorems and will, consequently, be left to the care of the reader.

13. A system of hypergeodesics associated with an arbitrary congruence of the first kind. Consider the transversal surfaces $S_{\rho_{\lambda}}$ and $S_{\sigma_{\lambda}}$ of the $R_{\lambda}$-associate of an arbitrary congruence $\Gamma$ of the first kind. The planes $\pi_{\nu}$ and $\pi_{\rho_{\lambda}}$ which are tangent at $y, \rho_{\lambda}$ and $\sigma_{\lambda}$ to the surfaces $S, S_{\rho_{\lambda}}$, and $S_{\sigma_{\lambda}}$, respectively, have in general the unique point in common which we denote by $P^{(\lambda)}$.

THEOREM 13.1. If the tangent at a general point $y$ to a curve $C_{\lambda}$ of $S$ contains the point $P^{(\lambda)}$ associated with an arbitrary congruence $\Gamma$ at the point $y$, the curve is a hypergeodesic of a system which we shall call the $\Gamma$-geodesics.

The curvilinear differential equation for the $\Gamma$-geodesics may easily be found to be

$$
d^{2} v / d u^{2}=\left(b_{u} / b+\beta+\beta_{(a)}\right) d v / d u-\left(a_{v}^{\prime} / a^{\prime}+\alpha+\alpha_{(a)}\right)(d v / d u)^{2},
$$

where $\beta, \alpha$ are the functions identified with the congruence $\Gamma$, and $\beta_{(a)}, \alpha_{(a)}$ are those identified with the asymptotic associate of the congruence $\Gamma$.

To obtain equation (13.1) we express the condition that the tangent $y P^{(\lambda)}$ shall have the direction $d v / d u=\lambda$. Since the point $P^{(\lambda)}$ is the intersection of the line joining the points $\rho_{\lambda},\left(\sigma_{\lambda}\right)_{(a)}$ with the line joining the points $\sigma_{\lambda},\left(\rho_{\lambda}\right)_{(a)}$, where $\left(\rho_{\lambda}\right)_{(a)}$ and $\left(\sigma_{\lambda}\right)_{(a)}$ determine the asymptotic associate of the line $l_{\lambda}$ joining $\rho_{\lambda}$ and $\sigma_{\lambda}$, the general coordinates for $P^{(\lambda)}$ may be found to be given by

$$
\left[\alpha_{\lambda}-\left(\alpha_{\lambda}\right)_{(a)}\right] y_{u}+\left[\beta_{\lambda}-\left(\beta_{\lambda}\right)_{(a)}\right] y_{v},
$$

in which

$$
\begin{aligned}
\alpha_{\lambda} & =\alpha+2 a^{\prime} \lambda, \quad \beta_{\lambda}=\beta+2 b / \lambda, \\
\left(\alpha_{\lambda}\right)_{(a)} & =-\left(f+\beta_{u}+[2 b / \lambda]_{u}+\beta^{2}+4 b \beta / \lambda+4 b^{2} / \lambda^{2}\right) / 2 b, \\
\left(\beta_{\lambda}\right)_{(a)} & =-\left(g+\alpha_{v}+\left[2 a^{\prime} \lambda\right]_{v}+\alpha^{2}+4 a^{\prime} \alpha \lambda+4 a^{\prime 2} \lambda^{2}\right) / 2 a^{\prime} .
\end{aligned}
$$

The direction of the tangent $y P^{(\lambda)}$ is therefore given by

$$
d v / d u=\left[\beta_{\lambda}-\left(\beta_{\lambda}\right)_{(a)}\right] /\left[\alpha_{\lambda}-\left(\alpha_{\lambda}\right)_{(a)}\right] .
$$

On setting the right member of this equation equal to $\lambda$, simplifying and putting $\lambda=d v / d u, \lambda_{u}+\lambda \lambda_{v}=d^{2} v / d u^{2}$, we obtain equation (13.1).

The cusp-axis of the $\Gamma$-geodesics at the point $y$ joins $y$ to a point $z$ given by (1.3) in which

$$
\alpha=-a_{v}^{\prime} / 2 a^{\prime}-\left(\alpha+\alpha_{(a)}\right) / 2, \quad \beta=-b_{u} / 2 b-\left(\beta+\beta_{(a)}\right) / 2 .
$$


We state now two interesting theorems; the first characterizes the directrix of the first kind, of Wilczynski, and the second characterizes the edge of the first kind, of Green. The duals of these theorems characterize the corresponding lines of the second kind. The proofs of the theorems and the statements of their duals will be left to the care of the reader.

THEOREM 13.2. The reciprocal of the cusp-axis at $y$ of the $\Gamma$-geodesics is the directrix of the first kind, of Wilczynski, if, and only if, the reciprocal of the projective normal separates harmonically the tangent $t_{(a)}$ with respect to the lines $l$ and $l_{(a)}$ of the congruences $\Gamma$ and $\Gamma_{(a)}$, respectively.

THEOREM 13.3. The reciprocal of the cusp-axis of the $\Gamma$-geodesics at $y$, the harmonic conjugate of $t_{(a)}$ with respect to the lines $l$ and $l_{(a)}$, and the first edge of Green, intersect in the same point, which we denote by $E$.

By choosing two congruences $\Gamma_{1}$ and $\Gamma_{2}$ of the first kind, having associated with them distinct points $E_{1}$ and $E_{2}$, we determine the first edge of Green as the line joining $E_{1}$ and $E_{2}$.

E. СECH

\section{REFERENCES}

1. L'intorno d'un punto d'una superficie considerato dal punto di vista proiettivo, Annali, di Matematica (3), vol. 31 (1922), pp. 191-206.

G. FUBini AND E. ČECH

1. Introduction à la Géométrie Projective Différentielle des Surfaces, Paris, Gauthier-Villars, 1931.

G. M. GREeN

1. Memoir on the general theory of surfaces and rectilinear congruences, these Transactions, vol. 20

E. P. LANE (1919), pp. 79-153.

1. The correspondence between the tangent plane of a surface and its point of contact, American P. SPERRY Journal of Mathematics, vol. 48 (1926), pp. 204-214.

1. Properties of a certain projectively defined two parameter family of curves on a general surface, American Journal of Mathematics, vol. 40 (1918), pp. 213-224.

E. J. WrLczYNSKI

1. Projective differential geometry of curved surfaces (second memoir), these Transactions, vol. 9 (1908), pp. 79-120.

2. Geometrical significance of isothermal conjugacy of a net of curves, American Journal of Mathematics, vol. 42 (1920), pp. 211-221.

UNIVERSITY OF KANSAS,

LAWRENCE, Kan. 